\title{
Cluster structures, ellipsoidal shapes and nuclear molecules in light A $=$ 12-50 nuclei
}

\author{
A. V. Afanasjev ${ }^{1,2, *}$ \\ ${ }^{1}$ Department of Physics and Astronomy, Mississippi State University, Mississippi 39762, USA \\ ${ }^{2}$ Yukawa Institute for Theoretical Physics, Kyoto University, Kyoto 606-8502, Japan
}

\begin{abstract}
The transition from cluster structures to extremely elongated ellipsoidal shapes and nuclear molecules in light $A=12-50(N \sim Z)$ nuclei has been studied within the framework of covariant density functional theory. Nodal structure of the occupied single-particle states plays a critical role in microscopic understanding of this transition. This is illustrated by the analysis of dominant types of single-particle density distributions and their evolution (from the bottom of nucleonic potential) with deformation and particle number. The microscopic mechanism of the transition from clustered structures to ellipsoidal shapes and nuclear molecules and between them is discussed.
\end{abstract}

\section{Introduction}

Light nuclei are characterized by the variety of the types of nucleonic configurations with different nuclear shapes. Ellipsoidal shapes are the most abundant ones, but there are also cluster structures and nuclear molecules. Cluster and extremely deformed structures in light nuclei have attracted a considerable interest (both experimental and theoretical) in recent years (see, e.g., Refs. [1-8]). Different models have been applied to the description of such structures. Cluster models provide an important insight into cluster dynamics of nucleus but are limited by initial assumptions about clusters and impossibility to describe many shell model configurations. Note that the cluster description does not correspond to clearly separated $\alpha$ particles, but generates the mean-field states largely by antisymmetrization [9].

Density functional theory (DFT) is alternative framework which has been applied to the description of the variety of nuclear structures in light nuclei $[3,7,10-15]$. It does not assume the existence of cluster structures and allows simultaneous treatment of cluster and mean-fieldtype states. In this framework, the formation of clusters proceeds from microscopic single-nucleon degrees of freedom via many-body correlations.

In this manuscript, a short review of recent results obtained for light $A=12-50$ nuclei within the covariant density functional theory (CDFT; see Ref. [16]) is presented ${ }^{1}$. A special attention is paid to the discussion of the microscopic mechanism of the transition between different nuclear shapes.

\footnotetext{
*e-mail: Anatoli.Afanasjev@gmail.com

${ }^{1}$ For results obtained in non-DFT frameworks for light $A \leq 20$ nuclei I refer the readers to recent extensive review [8].
}

\section{Nuclear shapes in the $N \sim Z$ and $A=12-50$ mass region}

Over recent years a number of the studies of the cluster structures, ellipsoidal shapes and nuclear molecules has been undertaken both at spin zero and at high spins in the CDFT framework $[3,7,11,13-15,17-19]$. The results for ground states presented in Refs. [3, 11, 13] are not discussed here since extremely deformed shapes which become yrast or near yrast at high spin are in the focus of the present manuscript. Figure 1 shows both the part of nuclear chart in which exotic shapes have been studied at high spin in the CDFT framework and most interesting examples of exotic nuclear shapes. These studies have been performed using cranked relativistic mean field (CRMF) approach $[20,21]$ in which the pairing correlations are neglected. The calculated configurations are labeled by shorthand $\left[n_{1}\left(n_{2}\right)\left(n_{3}\right), p_{1}\left(p_{2}\right)\left(p_{3}\right)\right]$ labels [7], where $n_{1}, n_{2}$ and $n_{3}$ are the number of neutrons in the $N=3$, 4 and 5 intruder/hyperintruder/megaintruder orbitals and $p_{1}, p_{2}$ and $p_{3}$ are the number of protons in the $N=3,4$ and 5 intruder/hyperintruder/megaintruder orbitals. The labels $n_{i}$ and $p_{i}$ are omitted in the labeling of the configurations if the respective orbitals are not occupied. Note that proton and neutron total and single-particle densities are extremely similar because of the $N \approx Z$ character of the nuclei under study [15]. In addition, calculated configurations of interest have nearly axial shapes [7, 14, 17, 18].

A linear chain of three $\alpha$-clusters, leading to "rodshaped" nucleus, is calculated in ${ }^{12} \mathrm{C}$ in Refs. [13, 17]. Its density distribution is shown in left bottom corner of Fig. 1. A number of similar $\alpha$-cluster configurations has been found in neighboring $\mathrm{C}$ nuclei (see Refs. [17, 22].) Another example of rod-shaped nucleus is linear chain configuration of four $\alpha$-clusters in ${ }^{16} \mathrm{O}$ investigated using Skyrme cranked Hartree-Fock method in Ref. [23] 


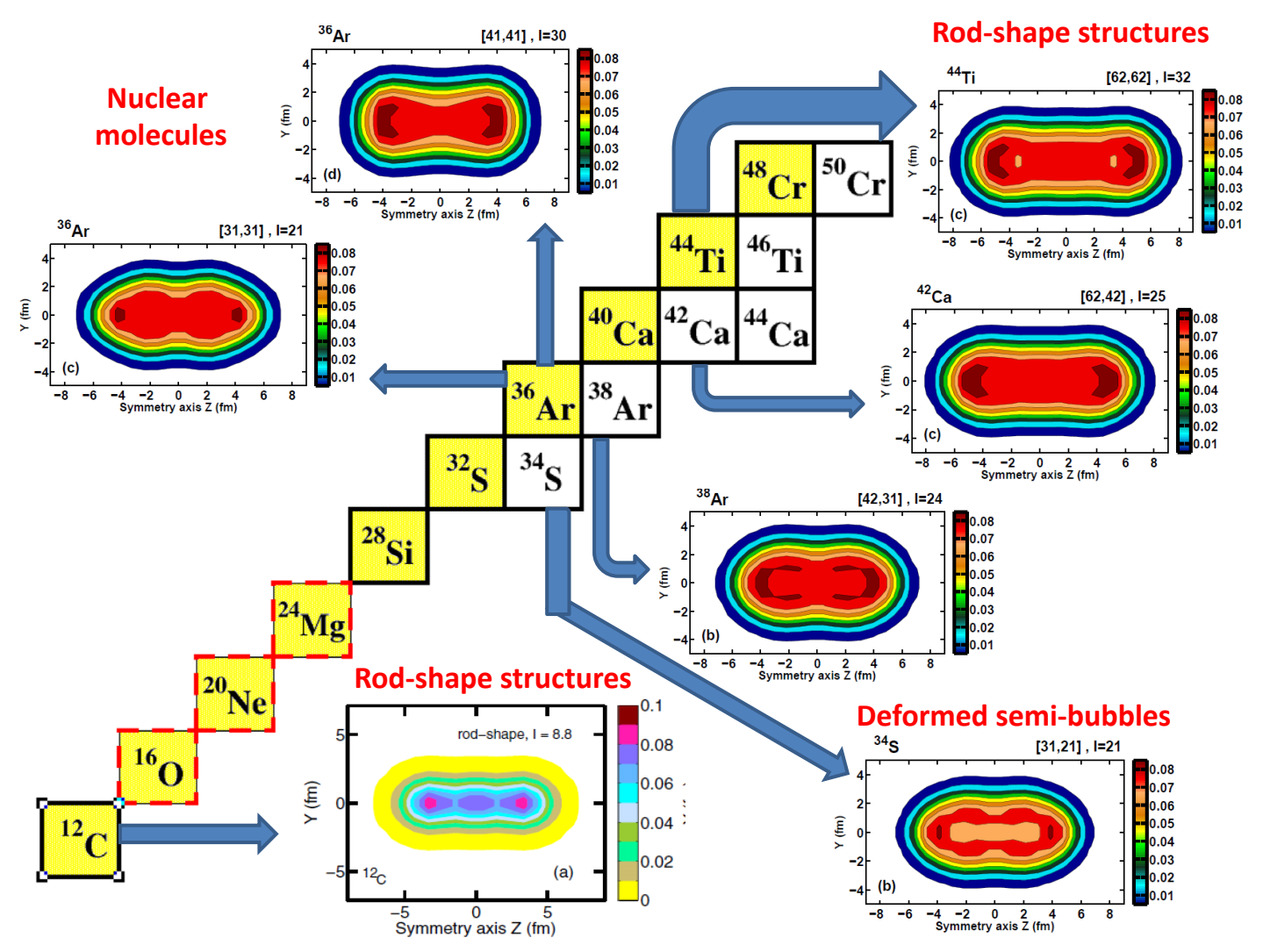

Figure 1. (Color online) The nuclei in which extremely deformed and rod-shaped structures have been studied in the DFT calculations during last decade. The boxes with black lines are used for the nuclei which have been studied in the CDFT framework by us in Refs. $[7,15,18,19]$. Yellow background is used for the nuclei with $N=Z$. Note that rod-shaped structures in the ${ }^{16} \mathrm{O}$ and ${ }^{24} \mathrm{Mg}$ nuclei (indicated by boxes with red dashed lines) and in the ${ }^{12} \mathrm{C}$ nucleus have been studied in the covariant and Skyrme DFT frameworks in Refs. $[5,14,17,23]$. Neutron density distributions of several nuclei are plotted in order to indicate the variety of reflection symmetric nuclear shapes possible in this mass region (see text for details). Based on the results presented in Refs. [7, 15, 18, 19]. Note that the density plot for ${ }^{12} \mathrm{C}$ is taken from Ref. [15]: as compared with other density plots it has different colormap but keeps the units of length in the $y$ and $z$ directions consistent with other density plots. The density plots provide also information on the configurations of these states and spin values at which they were calculated (see Refs. [15, 18] for more details).

and CRMF approach in Ref. [14]. However, as shown in Fig. 1 with increasing mass number the formation of the $\alpha$ cluster structures becomes significantly less likely and at extreme deformations there is a competition of the nucleonic configurations with ellipsoidal (see Ref. [7]) and rodshaped (the [62,62] configuration of ${ }^{44} \mathrm{Ti}$ and the $[62,42]$ configuration of ${ }^{42} \mathrm{Ca}$ in Fig. 1) shapes. Moreover, more exotic shapes such as nuclear molecules (the [31,31] and [41,41] configurations of ${ }^{36} \mathrm{Ar}$ in Fig. 1) and deformed semi-bubbles (the $[31,21]$ configuration of ${ }^{34} \mathrm{~S}$ in Fig. 1) are formed. A specific feature of the nuclear molecules is the existence of two fragments connected by the neck while deformed semi-bubbles are characterized by the central (elongated in shape) depression in the density distribution. It is clear that there should be some microscopic mechanism which dictates the transition from one type of shape to another one and the evolution/dominance of available shapes as a function of particle numbers. This mechanism is discussed in next section.

\section{Underlying single-particle structure and the role of nodal structure of the single-particle wave functions}

Many features of light nuclei depend sensitively on the underlying single-particle structure. The wave function $\Psi_{\left[N n_{z} \Lambda\right] \Omega}$ of the single-particle state denoted by the Nilsson quantum number $\left[N n_{z} \Lambda\right] \Omega$ can be expanded into the basis states $\left|N^{\prime} n_{z}^{\prime} \Lambda^{\prime} \Omega^{\prime}\right\rangle$ by

$$
\Psi_{\left[N n_{z} \Lambda\right] \Omega}=\sum_{N^{\prime} n_{z}^{\prime} \Lambda^{\prime} \Omega^{\prime}} c_{N^{\prime} n_{z}^{\prime} \Lambda^{\prime} \Omega^{\prime}}\left|N^{\prime} n_{z}^{\prime} \Lambda^{\prime} \Omega^{\prime}\right\rangle,
$$

which leads to the single-particle density $\rho_{\left[N n_{z} \Lambda\right] \Omega}$ given by

$$
\rho_{\left[N n_{z} \Lambda\right] \Omega}=\sum_{N^{\prime} n_{z}^{\prime} \Lambda^{\prime} \Omega^{\prime}} c_{N^{\prime} n_{z}^{\prime} \Lambda^{\prime} \Omega^{\prime}}^{2}\left\langle N^{\prime} n_{z}^{\prime} \Lambda^{\prime} \Omega^{\prime} \mid N^{\prime} n_{z}^{\prime} \Lambda^{\prime} \Omega^{\prime}\right\rangle .
$$

Here, the basis states are characterized by principal quantum number $N^{\prime}$, the number $n_{z}^{\prime}$ of nodes in the axial direction ( $z$-direction) and the projections of orbital $\left(\Lambda^{\prime}\right)$ and total $\left(\Omega^{\prime}\right)$ single-particle angular momenta on the axis of 


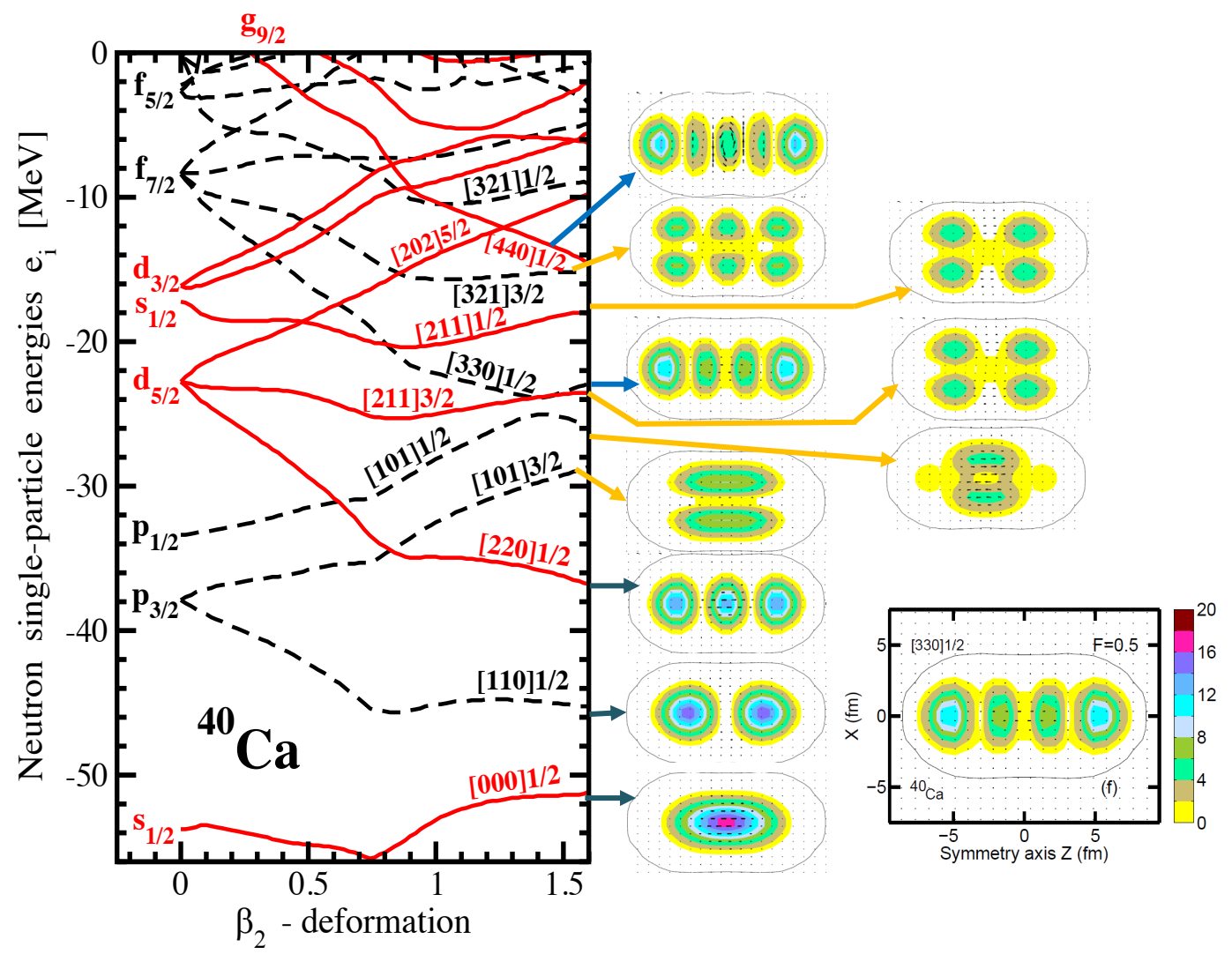

Figure 2. (Color online) Left panel: Single-particle energies, i.e., the diagonal elements of the single-particle Hamiltonian $\mathcal{H}$ in the canonical basis [24], for the lowest in total energy solution in the nucleus ${ }^{40} \mathrm{Ca}$ calculated as a function of the quadrupole equilibrium deformation $\beta_{2}$. The upper value of $\beta_{2}$ approximately corresponds to quadrupole deformations typical for megadeformed configurations in this nucleus. The calculations are performed in axial relativistic Hartree-Bogoliubov framework [25] using the NL3 ${ }^{*}$ covariant energy density functional [26]. The orbitals are labelled by means of asymptotic quantum numbers (Nilsson labels) $\left[N n_{z} \Lambda\right] \Omega$. Right panels: Single-neutron density distributions due to the occupation of indicated Nilsson states. The box in right bottom corner exemplifies physical dimensions of the nucleus as well as the colormap used for single-particle densities. Other density plots are reduced down to the shape and size of the nucleus which is indicated by black solid line corresponding to total neutron density line of $\rho=0.001 \mathrm{fm}^{-3}$. The colormap shows the densities as multiplies of $0.001 \mathrm{fm}^{-3}$; the plotting of the densities starts with yellow color at $0.001 \mathrm{fm}^{-3}$. The densities are based on the results of Ref. [15].

symmetry. The sum in these equations runs over all allowable combinations of the quantum numbers $N^{\prime}, n_{z}^{\prime}, \Lambda^{\prime}$ and $\Omega^{\prime}$.

The weights $c_{N^{\prime} n_{z}^{\prime} \Lambda^{\prime} \Omega^{\prime}}^{2}$ define the contributions of the basic states $\left|N^{\prime} n_{z}^{\prime} \Lambda^{\prime} \Omega^{\prime}\right\rangle$ into the single-particle density. Table 1 shows that in the nuclei/deformations of interest the single-particle wave functions are dominated by a single very large component (basis state) which in turn will define the spatial distribution of the single-particle density. This domination is especially pronounced for the singleparticle orbitals located at the bottom of the nucleonic potential; the rotation somewhat reduces this dominance but still preserves it (see Ref. [15]).

These features are extremely important for an understanding of the $\alpha$-clusterization and the evolution of extremely elongated shapes in light nuclei since these basis states define the nodal structure of the wave functions of the single-particle states and their density distributions. Three basic types of single-particle density distributions, namely, spheroidal/elipsoidal shapes, doughnut and ring shapes, play an important role in forming the nu- clear shapes at large elongation. In addition, there is very simple connection between the type of single-particle density distribution and the Nilsson label of the state [15]:

(1) Axial or nearly axially symmetric spheroidal/elipsoidal like density clusters are formed by the Nilsson states with the $[N N 0] 1 / 2$ quantum numbers. This is because dominant basis states of these Nilsson states have no nodes in radial direction and $n_{z}=N$ nodes in axial direction. Density clusters with highest densities are located in polar regions for $N>0$.

(2) Doughnut density distributions are formed by the Nilsson $[N 01] \Omega$ states because the densities of their dominant basis states have one node in radial direction and no nodes in axial direction.

(3) Multiply (two for $n_{z}=1$ and three for $n_{z}=2$ ) ring shapes for $N=2$ and 3 are formed by Nilsson states with the structure $[N, N-1,1] \Omega$.

These features are the consequences of the dominance of the wave function by a single basis state where only two 


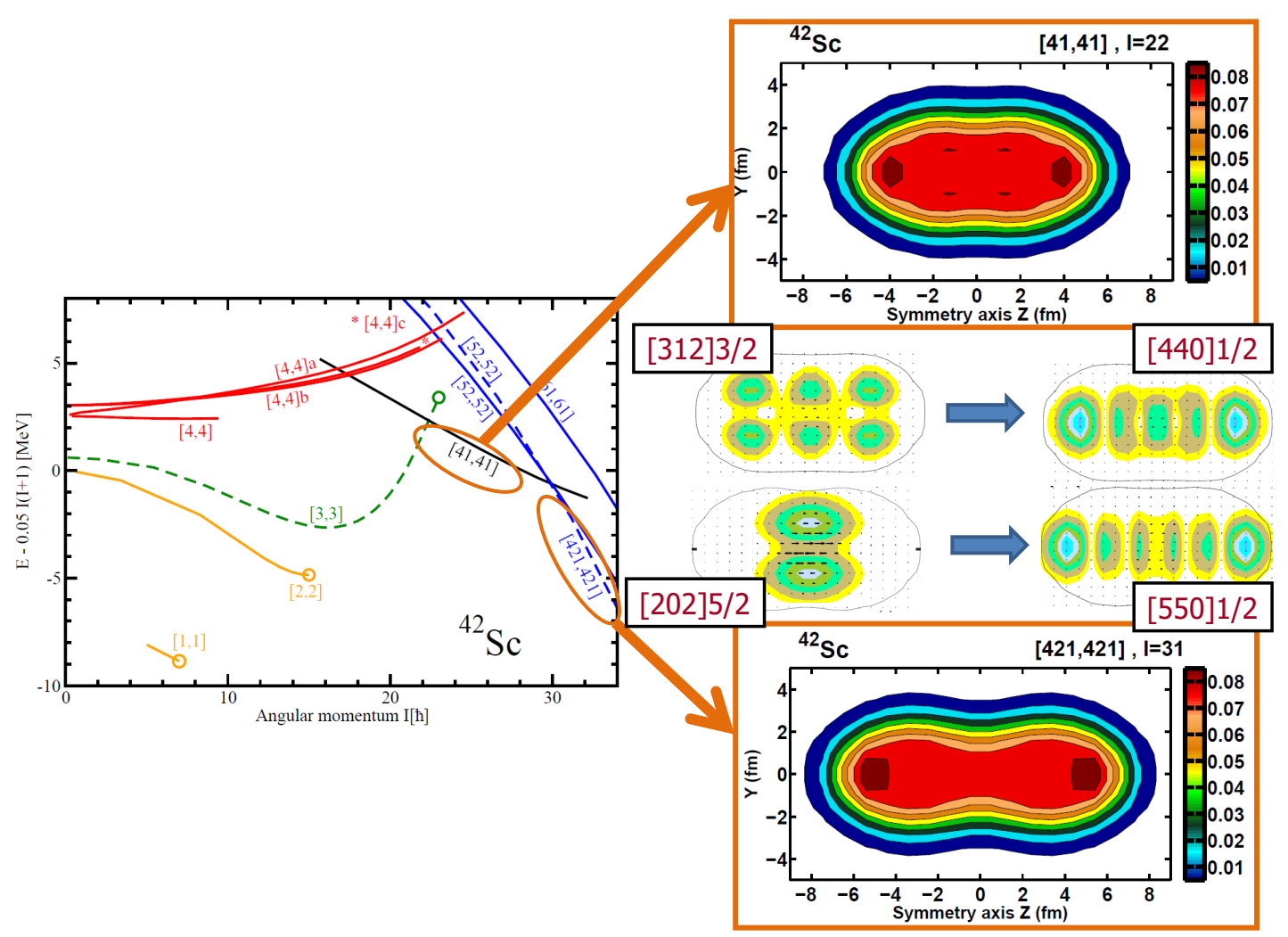

Figure 3. (Color online) Left panel: Energies of the calculated configurations in ${ }^{42} \mathrm{Sc}$ relative to a smooth liquid drop reference $A I(I+1)$, with the inertia parameter $A=0.05$ (right column, top and bottom panels). The self-consistent proton density $\rho_{p}(y, z)$ as a function of $y$ and $z$ coordinates for the indicated configurations at specified spin values. The equidensity lines are shown in steps of $0.01 \mathrm{fm}^{-3} \mathrm{starting}$ from $\rho_{p}(y, z)=0.01 \mathrm{fm}^{-3}$ (right column, middle). The single-particle densities of the orbitals by which the [41,41] and [421,421] configurations differ. The direction of particle-hole excitations by which the [421,421] configuration is created from the [41,41] one (see text for more details) is shown by large dark blue arrows. Based on the results of Refs. [7, 15].

types of the basis states are important, namely, the $|N, N, 0\rangle$ and $|N, N-1,1\rangle$ states. The density distributions of the basis states with $|N N 0\rangle$ will be axially symmetric with the maximum of density located at the axis of symmetry. The basis states with $|N, N-1,1\rangle$ structure will have a zero density at the axis of symmetry.

Figure 2 shows the evolution of the energies of the single-particle states with quadrupole deformation in ${ }^{40} \mathrm{Ca}$ nucleus and the single-particle densities of these states at the deformations typical for megadeformed (MD) configurations in this nucleus. One can see that these density distributions show all the features discussed above. In addition, Figure 2 clearly indicates the importance of the deformation which has two critical effects. First, it leads to the formation of density clusters with specific nodal structure (remember, the spherical states from which deformed states originate have spherical density distributions) and to the separation of the clusters in space. This is especially critical for the formation of the $\alpha$-clusters.

Second, it lowers the energies of the Nilsson states of the $[N N 0] 1 / 2$ type which favor the $\alpha$-clusterization and leads to the situation when all occupied Nilsson states have this type of structure. Indeed, the occupation of the [000]1/2, [110]1/2 and [220]1/2 proton and neutron or- bitals is responsible for triple $\alpha$-cluster structure in ${ }^{12} \mathrm{C}$ (see Fig. 1 and Refs. [15, 17]). Interestingly enough the occupation of these states in the MD $[42,42]$ configuration of ${ }^{40} \mathrm{Ca}$ still forms triple $\alpha$-cluster structure (see Ref. [15]). However, the total wave function of this configuration is more complex due to the presence of other single-particle orbitals which do not favor the formation of $\alpha$-cluster structures. The formation of the chain of four $\alpha$-clusters will require the occupation of four lowest states of the $[N N 0] 1 / 2$ type. Figure 2 clearly shows that such a situation is achievable by modest increase of quadrupole deformation beyond $\beta_{2}=1.6$ leading to the lowering of the [330]1/2 state below the [101]3/2 state. Such structure has been studied in the ${ }^{16} \mathrm{O}$ nucleus in the Skyrme DFT and CDFT in Refs. [14, 23]. However, the formation of the chain of five $\alpha$-clusters is more energetically expensive since it requires substantial increase of quadrupole deformation leading to the lowering of the [440]1/2 state below the [321]3/2, [211]1/2, [211]3/2, [101]1/2 and [101]3/2 states (see Fig. 2). Even higher energy cost and higher deformation are required for the formation of the chain of six $\alpha$-clusters.

It is necessary to recognize that these $\alpha$-cluster chains are built by multiply particle — multiply hole excita- 
Table 1. The squared amplitudes $c_{N^{\prime} n_{z}^{\prime} \Lambda^{\prime} \Omega^{\prime}}^{2}$ of two largest components of the wave functions of the neutron single-particle states occupied in the MD [42,42] configuration of ${ }^{40} \mathrm{Ca}$ at spin zero. The states are shown from the bottom of nucleonic potential in the same sequence as they appear in the routhian diagram of Fig. $2 b$ in Ref. [15] at frequency $\Omega_{x}=0.0 \mathrm{MeV}$

\begin{tabular}{l|l}
\hline State & Wave function \\
\hline$[000] 1 / 2$ & $92.7 \%|000,1 / 2\rangle+6.7 \%|220,1 / 2\rangle+$ \\
{$[110] 1 / 2$} & $89.6 \%|110,1 / 2\rangle+9.6 \%|330,1 / 2\rangle+$ \\
{$[220] 1 / 2$} & $85.1 \%|220,1 / 2\rangle+6.5 \%|000,1 / 2\rangle+$ \\
{$[101] 3 / 2$} & $92.9 \%|101,3 / 2\rangle+6.7 \%|321,3 / 2\rangle+$ \\
{$[330] 1 / 2$} & $75.7 \%|330,1 / 2\rangle+8.5 \%|110,1 / 2\rangle+$ \\
{$[211] 3 / 2$} & $92.5 \%|211,3 / 2\rangle+6.9 \%|431,3 / 2\rangle+$ \\
{$[101] 1 / 2$} & $90.2 \%|101,1 / 2\rangle+5.0 \%|330,1 / 2\rangle+$ \\
{$[211] 1 / 2$} & $81.4 \%|211,1 / 2\rangle+9.6 \%|431,1 / 2\rangle+$ \\
{$[321] 3 / 2$} & $85.7 \%|321,3 / 2\rangle+6.1 \%|101,3 / 2\rangle+$ \\
{$[440] 1 / 2$} & $77.4 \%|440,1 / 2\rangle+10.7 \%|211,1 / 2\rangle+$ \\
{$[550] 1 / 2$} & $79.3 \%|550,1 / 2\rangle+6.8 \%|541,1 / 2\rangle+$ \\
\hline
\end{tabular}

tions with respect of the ground state configuration. As a result, they are located at high excitation energies (see Refs. $[14,15,17,23])$ and thus are very difficult to observe in experiment. The low-energy excited states in the mass region of interest are build at either spherical or normal/high deformation or superdeformation. Figure 2 clearly indicates that at these deformations nucleonic configurations are typically build by the mixture of the $|N, N, 0\rangle$ and $|N, N-1,1\rangle$ states and the latter states become more abundant with the increase of particle number. Moreover, at low deformations the nodal structure of the single-particle density distributions becomes affected by increased mixing between the basis states and the separation between the density clusters becomes reduced so that separate $\alpha$-clusters could not be formed. As a result, apart of well-known two $\alpha$-cluster structure in ${ }^{8} \mathrm{Be}$ and similar structures in neighboring nuclei [27], the possibilities for the formation of $\alpha$-clusters at such deformations are substantially reduced. In reality, calculated shapes of absolute majority of nucleonic configurations at such deformations are ellipsoidal.

The situation is similar at megadeformation $\left(\beta_{2} \sim 1.6\right)$ for particle numbers above 6 (see Fig. 2). The $|N, N-1,1\rangle$ states with doughnut or multiply-ring single-particle densities become more abundant than the $|N, N, 0\rangle$ states. As a result, the contribution of the states, favoring $\alpha$ clusterization, into the structure of the total wave function decreases and ellipsoidal nuclear shapes as well as nuclear molecules become dominant species of nuclear shapes at these particle numbers.

\section{Transition from ellipsoidal shapes to nuclear molecules by means of particle-hole excitations}

The results of systematic investigations of nuclear shapes at high spin performed in Ref. [7] show interesting examples of the coexistence of elipsoidally shaped structures and nuclear molecules in the same nucleus at com- parable spins and frequently at similar elongations of nuclear shape. It turns out that the configurations of these two types of the shapes are connected by characteristic particle-hole excitations (see Ref. [15]). In order to build nuclear molecules from typical ellipsoidal density distributions one has to move the matter from the neck (equatorial) region into the polar regions of the nucleus. This can be achieved by means of specific particle-hole excitations which move the particles from (preferentially) doughnut type orbitals or from the orbitals which have a density ring in an equatorial plane into the orbitals (preferentially of the $[N N 0] 1 / 2$ type) building the density mostly in the polar regions of the nucleus.

This microscopic mechanism of the creation of nuclear molecule is illustrated in Fig. 3 on the example of the hyperdeformed (HD) [41,41] and MD [421,421] configurations in ${ }^{42} \mathrm{Sc}$ nucleus. The [41,41] configuration is yrast in the spin range $I \approx 23-30 \hbar$ and it has ellipsoidal shape. At spin $I \sim 30 \hbar$, it is crossed by the MD [421,421] configuration which is yrast at $I \geq 30 \hbar$ (see left panel in Fig. 3). This configuration is nice example of nuclear molecule in which two fragments are connected by the neck. This transition is achieved in proton and neutron subsystems by the particle-hole excitations from the [202]5/2 and [321]3/2 orbitals into the [440]1/2(r=+i) and $[550] 1 / 2(r=+i)$ orbitals. At the deformations and rotational frequencies of interest, these orbitals are located in the vicinity of each other (see Figs. 3 and 4 in Ref. [7]) and this explains why the total energies of these two configurations are comparable (see left panel in Fig. 3). The [202]5/2 orbital has doughnut type density distribution and [321]3/2 has triple ring density distribution (see middle of right column in Fig. 3); these two orbitals are occupied in the HD [41,41] configuration of ${ }^{42} \mathrm{Sc}$. On the contrary, the $[440] 1 / 2(r=+i)$ and $[550] 1 / 2(r=+i)$ orbitals have the largest and most dense density clusters in the polar regions of the nucleus (see middle of right column in Fig. 3); these two orbitals are occupied in the MD $[421,421]$ configuration. Thus, particle-hole excitations $[202] 5 / 2+[321] 3 / 2 \rightarrow[440] 1 / 2(r=+i)+[550] 1 / 2(r=+i)$ performed both in proton and neutron subsystems move the matter from the neck (equatorial) region into the polar regions of the nucleus and form the nuclear molecule.

There are other examples of nuclear molecules in the region of interest. These are the MD configurations [31,31] and [41,41] in ${ }^{36} \mathrm{Ar}$ (see Fig. 1) and MD $[42,42]$ configuration in ${ }^{40} \mathrm{Ca}$ (see Ref. [7]). Another well known case of molecular structure in this mass region is the superdeformed configuration $[2,2]$ in ${ }^{32} \mathrm{~S}[7,9,28]$; according to Refs. $[9,28]$ the wave function of this configuration contains significant admixture of the molecular ${ }^{16} \mathrm{O}+{ }^{16} \mathrm{O}$ structure. Similar to above discussed case of ${ }^{42} \mathrm{Sc}$, they are created by respective particle-hole excitations. For example, nuclear molecule in the MD [31,31] configuration of ${ }^{36} \mathrm{Ar}$ is created from the HD $[4,4]$ configuration, which has ellipsoidal density distribution (see Fig. 24b in Ref. [7]), by particle-hole excitations from the [321]3/2 orbital (which has triple ring density distribution (see Fig. 2) into the [440]1/2(r=-i) orbital. 


\section{Conclusions}

The competition of different types of nuclear shapes (cluster, ellipsoidal and nuclear molecules) in light $A=12-50$ nuclei and its evolution with mass number have been analyzed in covariant density functional theory. The occupation of the [NN0]1/2 single-particle states favors $\alpha$ clusterization because of specific features of the nodal structure of their single-particle density distributions. Density clusters formed by these states are well localized, have spheroidal or somewhat ellipsoidal density distribution and are located on the axis of symmetry of the nucleus. On the contrary, the $[N, N-1,1] \Omega$ states have doughnut (for $N=1$ ) and multiply-ring-type (for $N>1$ ) density distributions. Thus, the occupation of such states favors ellipsoidal shapes or nuclear molecules but disfavors $\alpha$-clusterization since such density distributions are incompatible with $\alpha$-clusters. The abundance/dominance of such states increases with particle number and this explains the transition from frequent appearance of cluster structures in very light nuclei with $A \sim 12$ to the dominance of ellipsoidal shapes/nuclear molecules in the heavier $A>20$ nuclei.

I would like to express my gratitude to my collaborators, D. Ray and $\mathrm{H}$. Abusara, who contributed to the investigations presented here. This material is based upon work supported by the U.S. Department of Energy, Office of Science, Office of Nuclear Physics under Award no. DE-SC0013037.

\section{References}

[1] Y. Taniguchi, Y. Kanada-En'yo, M. Kimura, K. Ikeda, H. Horiuchi, E. Ideguchi, Phys. Rev. C 82, 011302(R) (2010)

[2] Y. Kanada-En'yo, Phys. Rev. C 85, 044320 (2012)

[3] J.-P. Ebran, E. Khan, T. Nikšić, D. Vretenar, Nature (London) 487, 341 (2012)

[4] Y. Taniguchi, Phys. Rev. C 90, 054308 (2014)

[5] Y. Iwata, T. Ichikawa, N. Itagaki, J.A. Maruhn, T. Otsuka, Phys. Rev. C 92, 011303(R) (2015)

[6] M. Kimura, T. Suhara, Y. Kanada-En'yo, Eur. Phys. J. A 52, 373 (2016)

[7] D. Ray, A.V. Afanasjev, Phys. Rev. C 94, 014310 (2016)
[8] M. Freer, H. Horiuchi, Y. Kanada-En'yo, D. Lee, U.G. Meißner, Rev. Mod. Phys. 90, 035004 (2018)

[9] J.A. Maruhn, M. Kimura, S. Schramm, P.-G. Reinhard, H. Horiuchi, A. Tohsaki, Phys. Rev. C 74, 044311 (2006)

[10] J.L. Egido, L.M. Robledo, Nucl. Phys. A 738, 31 (2004)

[11] P. Arumugam, B.K. Sharma, S.K. Patra, R.K. Gupta, Phys. Rev. C 71, 064308 (2005)

[12] P.-G. Reinhard, J.A. Maruhn, A.S. Umar, V.E. Oberacker, Phys. Rev. C 83, 034312 (2011)

[13] J.-P. Ebran, E. Khan, T. Nikšić, D. Vretenar, Phys. Rev. C 90, 054329 (2014)

[14] J.M. Yao, N. Itagaki, J. Meng, Phys. Rev. C 90, 054307 (2014)

[15] A.V. Afanasjev, H. Abusara, Phys. Rev. C 97, 024329 (2018)

[16] D. Vretenar, A.V. Afanasjev, G.A. Lalazissis, P. Ring, Phys. Rep. 409, 101 (2005)

[17] P.W. Zhao, N. Itagaki, J. Meng, Phys. Rev. Lett. 115, 022501 (2015)

[18] A.V. Afanasjev, D. Ray, J. Phys.: Conf. Ser. 863, 012502 (2017)

[19] A.V. Afanasjev, H. Abusara, S.E. Agbemava, Phys. Scr. 93, 034002 (2018)

[20] W. Koepf, P. Ring, Nucl. Phys. A 493, 61 (1989)

[21] A.V. Afanasjev, J. König, P. Ring, Nucl. Phys. A 608, 107 (1996)

[22] J.-P. Ebran, E. Khan, T. Nikšić, D. Vretenar, J. Phys. G: Nucl. Part. Phys. 44, 103001 (2017)

[23] T. Ichikawa, J.A. Maruhn, N. Itagaki, S. Ohkubo, Phys. Rev. Lett. 107, 112501 (2011)

[24] P. Ring, P. Schuck, The Nuclear Many-Body Problem (Springer-Verlag, Berlin, 1980)

[25] S.E. Agbemava, A.V. Afanasjev, D. Ray, P. Ring, Phys. Rev. C 89, 054320 (2014)

[26] G.A. Lalazissis, S. Karatzikos, R. Fossion, D.P. Arteaga, A.V. Afanasjev, P. Ring, Phys. Lett. B 671, 36 (2009)

[27] W. von Oertzen, M. Freer, Y. Kanada-En'yo, Phys. Rep. 432, 43 (2006)

[28] M. Kimura, H. Horiuchi, Phys. Rev. C 69, 051304(R) (2004) 